\title{
Differential expression of the CCN family members Cyr61, CTGF and Nov in human breast cancer
}

\author{
Wen G Jiang, Gareth Watkins, Oystein Fodstad ${ }^{1}$, Anthony Douglas-Jones ${ }^{2}$, \\ Kefah $\mathrm{Mokbel}^{3}$ and Robert E Mansel
}

\author{
Metastasis \& Angiogenesis Research Group, University of Wales College of Medicine, Cardiff University, Cardiff, UK \\ ${ }^{1}$ Cancer Research Institute, University of South Alabama, Mobile, AL, USA \\ ${ }^{2}$ Pathology, University of Wales College of Medicine, Cardiff University, Cardiff, UK \\ ${ }^{3}$ Department of Surgery, St George Hospital, London, UK \\ (Requests for offprints should be addressed to W G Jiang; Email: jiangw@cf.ac.uk)
}

\begin{abstract}
The CCN family members cysteine-rich 61 (Cyr61/CCN1), connective tissue growth factor (CTGF/ CCN2) and nephroblastoma over-expressed (Nov/CCN3) play diverse roles in cells, are known to regulate cell growth, adhesion, matrix production and migration and are involved in endocrineregulated pathways in various cell types. The role of these molecules in cancer remains controversial. In a cohort of 122 human breast tumours (together with 32 normal breast tissues) we have analysed the expression of all three CCN members at the mRNA and protein levels. Significantly higher levels of Cyr61 ( $P=0.02$ ), but low levels of CTGF and Nov, were seen in tumour tissues compared with normal tissues. Significantly raised levels of Cyr61 were associated with poor prognosis $(P=0.02)$, nodal involvement $(P=0.03)$ and metastatic disease $(P=0.016)$. Patients who died of breast cancer also had high levels of Cyr61. In contrast, CTGF in patients with poor prognosis $(P=0.021)$, metastasis ( $P=0.012)$, local recurrence $(P=0.0024)$ and mortality $(P=0.0072)$ had markedly reduced levels. Similar to CTGF, low levels of Nov were also seen in patients with poor prognosis and mortality and with significantly decreased survival ( $P=0.033$ and $P=0.0146$, respectively). This result was fully supported by immunohistochemical analysis of frozen sectioned tissues. While fibroblasts and endothelial cells generally expressed good levels of all three CCN proteins, highly invasive MDA MB 231 cells expressed lower levels of CTGF and Nov, but higher levels of Cyr61, than the less invasive MCF-7. It is concluded that members of the CCN family are differentially expressed and may play important but contrasting roles in the progressive nature of human breast cancer. While Cyr61 appears to act as a factor stimulating aggressiveness, CTGF and Nov may act as tumour suppressors.
\end{abstract}

Endocrine-Related Cancer (2004) 11 781-791

\section{Introduction}

The CCN family comprises cysteine-rich 61 (Cyr61/ CCN1), connective tissue growth factor (CTGF/CCN2) and nephroblastoma over-expressed (Nov/CCN3). Recently the Wnt-induced secreted proteins (WISPs) have been found to also belong to the CCN family and so WISP-1 has been named CCN4, WISP-2 is CCN5 and WISP-3 is CCN6. These proteins stimulate mitosis, adhesion, apoptosis, extracellular matrix production, growth arrest and migration of multiple cell types (for a recent review, see Brigstock 2003). Many of these activities probably occur through the ability of CCN proteins to bind and activate cell-surface integrins and intracellular signalling molecules including fibulin 1C, Notch 1, S100A4 and ion channels (Perbel 2004). Accumulating evidence supports a role for these factors in endocrine pathways and endocrine-related processes. Despite the progress in understanding the biology of these molecules in cells and signalling pathways, the role of the $\mathrm{CCN}$ members in cancer is far from clear and the results in the literature remain controversial.

Cyr61 is a known angiogenic factor (Babic et al. 1998, Mo et al. 2002), which acts probably by regulating pro- 
Table 1 Clinical information on patients in the study

\begin{tabular}{llr}
\hline Clinical Information & & $\boldsymbol{n}$ \\
\hline Nodal status & Negative & 65 \\
Grade & Positive & 55 \\
& Grade 1 & 23 \\
& Grade 2 & 41 \\
TNM staging & Grade 3 & 56 \\
& TNM-1 & 69 \\
& TNM-2 & 60 \\
Clinical outcome & TNM-3 & 7 \\
& TNM-4 & 4 \\
& Disease-free & 87 \\
& With metastasis & 6 \\
& With local recurrence & 5 \\
& Died of breast cancer & 19 \\
\hline
\end{tabular}

angiogenic integrins (Tsai et al. 2000, Leu et al. 2002). MCF-7 breast cancer cells, when transfected with Cyr61, acquire hormone independence and anti-oestrogen resistance. These cells become tumorigenic and invasive, accompanied by increased angiogenic activities. Cyr61 has been indicated in tumorigenesis and disease progression in breast tumour models (Tasi et al. 2002a). Cyr61 expression can be up-regulated by hormones such as progestin (Sampath et al. 2002), and agents including phorbol ester and vitamins D and E2 (Sampeth et al. 2001, Tsai et al. 2002b), but down-regulated by retinoic acid (Tsai et al. 2002b). The role for CTGF/CCN2 in cancer is far less clear. CTGF has been shown to affect cell-cycle progression, by up-regulating cyclin $\mathrm{A}$ and reducing $\mathrm{p} 27^{\mathrm{kip} 1}$ (Kothapalli and Grotendorst 2000). CTGF has been found to be highly expressed in proliferating endothelial cells and glioma cells (Pan et al. 2002). In breast cancer cells, CTGF mRNA was found to be absent from MCF-7. Transfection of MCF-7 with CTGF expression vector results in a high degree of apoptosis (Hishikawa et al. 1999). This has been supported partly by a recent study which shows that over-expression of CTGF in oral squamous cell carcinoma results in reduction in cell growth and tumorigenesis (Moritani et al. 2003). Nov/CCN3 is probably the least-studied CCN member. Nov has been indicated to stimulate the proliferation of fibroblasts (Liu et al. 1999). Over-expression of Nov in glioma cells results in cells with slower growth rate and low tumorigenicity (Gupta et al. 2001).

Thus, the role of the CCN family in clinical cancer is unclear and in many cases remains controversial. In the current study, we have examined the relationship between Cyr61, CTGF and Nov at the mRNA and protein levels in a cohort of human breast cancer, and studied the clinical outcomes. We report here that while Cyr61 was highly over-expressed in tumour tissues and was linked to the progressive nature of breast tumours, CTGF and Nov exhibited lower levels in breast tumours and were inversely linked to a poor prognosis, suggesting that differentially expressed CCN members may have clear contrasting roles in the development of human breast cancer.

\section{Materials and methods}

\section{Sample collection}

Human breast cancer cell lines MCF-7 and MDA MB 231, and human fibroblast cell line MRC-5, were purchased from the European Collection of Animal Cell Cultures (ECACC, Salisbury, Hants, UK). Human umbilical vein endothelial cells (HUVECs) were purchased from TCS Biologicals (Oxford, UK). Breast cancer tissues $(n=120)$ and normal background tissues $(n=32)$ were collected immediately after surgery and stored in a deep freeze until use. Patients were routinely followed clinically after surgery. The median follow-up period was 72 months for the current study. The presence of tumour cells in the collected tissues was verified by a consultant pathologist (A D-J), who examined Hematoxylin and Eosin (H\&E)-stained frozen sections. Details of the histology were obtained from pathology reports and have been given in Table 1 .

\section{Materials}

RNA-extraction kit and RT kit were obtained from AbGene, Guildford, Surrey, UK. PCR primers were designed using Beacon Designer (Palo Alto, CA, USA) and synthesized by Invitrogen (Paisley, Scotland, UK). Molecular-biology-grade agarose and DNA ladder were from Invitrogen. Mastermix for routine PCR and quantitative PCR was from AbGene. Goat anti-human Cyr61, CTGF and Nov antibodies were purchased from Santa Cruz Biotechnology (Santa Cruz, CA, USA).

\section{Tissue processing, RNA extraction and cDNA synthesis}

Frozen sections of tissues were cut at a thickness of 5$10 \mu \mathrm{m}$ and kept for immunohistochemistry and routine histology (Jiang et al. 2003a). A further 15-20 sections were homogenized using a hand-held homogenizer in icecold RNA extraction solution. The concentration of RNA was determined using a UV spectrophotometer. Reverse transcription was carried out using an RT kit with an anchored oligo-dT primer supplied by AbGene, using $1 \mu \mathrm{g}$ total RNA in a 96-well plate. The quality of cDNA was verified using $\beta$-actin primers.

\section{Quantitative analysis of CCN family members}

The transcript level of the $\mathrm{CCN}$ family members from the above-prepared cDNA was determined using a real-time 
quantitative PCR, based on the Amplifluor ${ }^{\mathrm{TM}}$ technology (Nazarenko et al. 1997), modified from a previously reported method (Jiang et al. 2003a, 2003b). Briefly, a pair of PCR primers was designed using the Beacon Designer software (version 2). To one of the primers (routinely the antisense primer in our laboratory), an additional sequence, known as the $\mathrm{Z}$ sequence (5'ACTGAACCTGACCGTACA-3'), which is complementary to the universal Z probe (Nazarenko et al. 1997; Intergen, Oxford, UK), was added. A Taqman detection kit for $\beta$-actin was purchased from Perkin-Elmer. The primers used were: Cyr61, 5'-GGGCTGGAATGCAA CTTC- $3^{\prime}$ and 5'-ACTGAACCTGACCGTACACGTT TTGGTAGATTCTGGAG-3' (spanning the third intron; GenBank accession no. AF307860); CTGF, 5'-GAGT GGGTGTGTGACGAG3' and 5'-ACTGAACCTGACC GTACAGGCAGT- TGGCTCTAATCATA-3' (spanning the fourth intron; NM_001901); and Nov, 5'-CTGTGAA CAAGAGCCAGAG- $\overline{3}^{\prime}$ and 5'-ACTGAACCTGA CCGTACACTTGAACTGCAGGTGGAT-3' (spanning positions 848-849; NM-002514). Primers used for quantitation of oestrogen receptor (ER) and ER- $\beta$ were as reported previously (Ye et al. 2003): ER, 5'-CCTAC TACCTGGAGAACGAG- $3^{\prime}$ and 5'-CTCTTCGGTCTT TTCGTATG-3'; and ER- $\beta$, 5'-AAAAGAATCATTCA ATGACA-3' and 5'-ATTAACACCTCCATCCAACA$3^{\prime}$. Cytokeratin-19 (CK19) was used for comparison of cellularity during the analysis and primers for CK19 were 5'-CAGGTCCGAGGTTACTGAC-3' and 5'-ACTGAA CCTGACCGTACACACTTTCTGCCAGTGTGTCTTC -3' (King et al. 2004, Parr and Jiang 2004).

The reaction was carried out using the following: Hotstart Q-master mix (Abgene), 10 pmol of specific forward primer, $10 \mathrm{pmol}$ of reverse primer which has the $\mathrm{Z}$ sequence, $100 \mathrm{pmol}$ of 6-carboxyfluorescein (FAM)tagged probe (Intergen), and cDNA from approximately 50 ng RNA (calculated from the starting RNA in the reverse transcriptase reaction). The reaction was carried out using IcyclerIQ ${ }^{\mathrm{TM}}$ (BioRad) which is equipped with an optic unit that allows real-time detection of 96 reactions, using the following conditions: $94^{\circ} \mathrm{C}$ for $120 \mathrm{~min}, 50$ cycles of $94^{\circ} \mathrm{C}$ for $150 \mathrm{~s}, 55^{\circ} \mathrm{C}$ for $400 \mathrm{~s}$ and $72^{\circ} \mathrm{C}$ for $200 \mathrm{~s}$ (Jiang et al. 2003b, 2003c, Parr and Jiang 2004). The levels of the transcripts were generated from an internal standard (Jiang et al. 2003a) that was simultaneously amplified with the samples, and are shown here in two ways: levels of transcripts based on equal amounts of RNA, and as a target/CK19 ratio.

\section{Immunohistochemical staining of the $\mathrm{CCN}$ family proteins}

Frozen sections of breast tumour and background tissue were cut at a thickness of $6 \mu \mathrm{m}$ using a cryostat (Jiang et al. 2003c). The sections were mounted on Super Frost Plus microscope slides, air dried and then fixed in a mixture of $50 \%$ acetone $/ 50 \%$ methanol. The sections were then placed in Optimax wash buffer for 5-10 min to rehydrate. Sections were incubated for $20 \mathrm{~min}$ in a $0.6 \%$ BSA blocking solution and probed with the primary antibody. Following extensive washings, sections were incubated for $30 \mathrm{~min}$ with the secondary biotinylated antibody (Multilink Swine anti-goat/mouse/rabbit immunoglobulin; Dako, Ely, Cambs, UK). Following washings, avidin-biotin complex (Vector Laboratories, Burlingame, CA, USA) was then applied to the sections followed by extensive washings. Diaminobenzidine chromogen (Vector Laboratories) was then added to the sections, which were incubated in the dark for $5 \mathrm{~min}$. Sections were then counterstained in Gill's haematoxylin and dehydrated in ascending grades of methanol before clearing in xylene and mounting under a cover slip. Cytoplasmic staining of the respective proteins was quantified using Optimas 6.0 software as we described previously (Davies et al. 2000, King et al. 2004) and is shown here as relative staining intensity.

Statistical analysis was carried out using MannWhitney U test, the Kruskal-Wallis test and survival analysis using Kaplan-Meier survival curves and Univariate analysis (SPSS11).

\section{Results}

\section{Expression of $\mathrm{CCN}$ members in breast tissues}

Using quantitative PCR, it was shown that Cyr61 displayed significantly higher levels in tumour tissues compared with normal tissues (Fig. 1). In contrast, levels of CTGF were significantly lower in tumour tissues than in normal tissues. The levels of Nov (CCN3) were also low in tumour tissues, but the difference was not statistically significant. When the levels of the transcripts were normalized by CK19, as revealed by the CCN:CK19 ratio (Fig. 1, inserts), similar trends were seen with respective molecules.

\section{Distribution of CCN members in different cell types}

In immunohistochemical analysis, strong Cyr61 staining was seen in both cancer cells and endothelial cells in tumour tissues (Fig. 2A \& B). In contrast, the staining of both cell types in normal tissues was visibly weaker than in cancer cells (Fig. 2A). The staining pattern of CTGF was in clear contrast with Cyr61, in that CTGF strongly stained normal epithelial cells, stromal cells and endothelial cells, but only weakly stained cancer cells (Fig. 2C \& D). However, it is noteworthy that matrix in tumour 

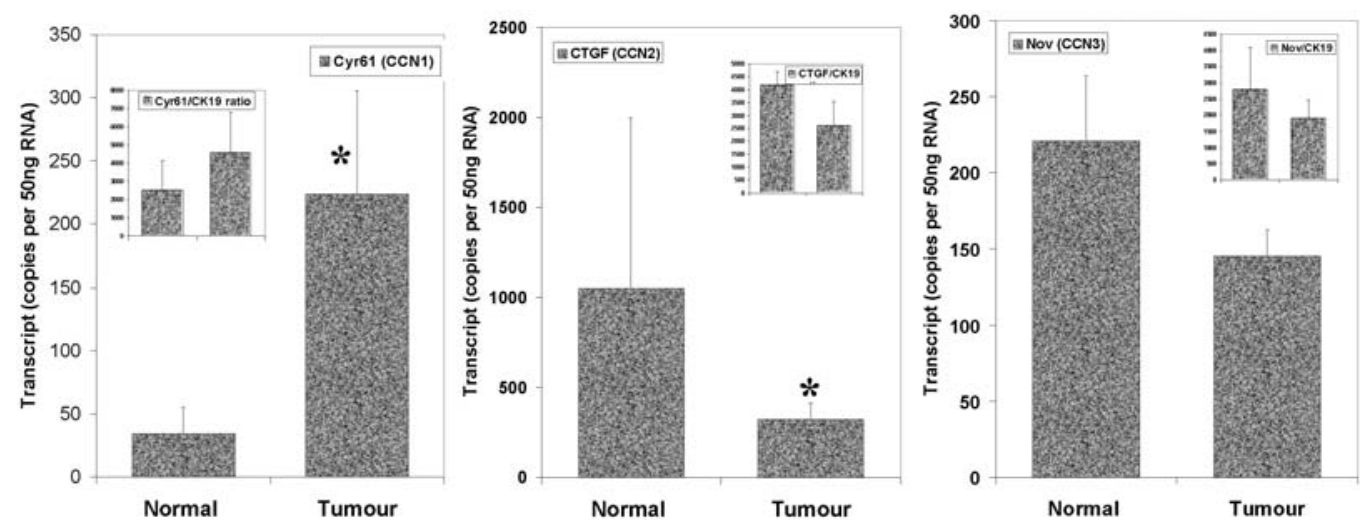

Figure 1 Levels of expression of Cyr61 (left-hand panel), CTGF (middle panel) and Nov (right-hand panel) in normal and tumour tissues. ${ }^{*} P<0.05$ compared with normal tissues. Inserts: Cyr61:CK19, CTGF:CK19 and Nov:CK19 ratios.

tissues exhibited some degree of staining. The pattern of Nov staining was similar to that of CTGF (Fig. 2E \& F). Fig. 3 shows the quantitative analysis of cytoplasmic staining of normal mammary epithelial cells and breast cancer cells, using image analysis. Protein levels of Cyr61 were significantly higher in cancer cells compared with normal mammary epithelial cells. In contrast, both CTGF and Nov proteins were seen at lower levels in breast cancer cells (Fig. 3, middle and right-hand panels).

To further verify the cell source of these molecules, we measured the levels of transcripts in highly aggressive MDA MB 231 and less-invasive MCF-7 breast cancer cells, MRC5 fibroblasts, and HECV and human umbilical vein endothelial cells (HUVEC) endothelial cells. Both fibroblasts and endothelial cells expressed high levels of all three members. The expression patterns of Cyr61 and those of CTGF and Nov were very different in breast cancer cell lines. The highly invasive MDA MB 231 cells expressed high levels of Cyr61, but relatively lower levels of CTGF and Nov, when compared with non-invasive $\mathrm{MCF}-7$ cells.

\section{Levels of CCN members and relationship with prognostic indices and nodal involvement}

The prognosis indices used here were 2-fold; nodal status and the Nottingham Prognostic Index (NPI; where NPI-1 represents patients with $\mathrm{NPI}<3.5$ and good prognosis, NPI-2 is $3.5-5.4$ with moderate prognosis, and NPI-3 is $>5.4$ with poor prognosis).

Significantly higher levels of Cyr61 were seen in NPI-3 tumours when compared with NPI-1 tumours $(P=0.02$; Fig. 4, left-hand panel). Although Cyr61 was higher in NPI-2 tumours, the difference between NPI-1 and NPI-2 was nonetheless not significant. In contrast to Cyr61, CTGF was significantly lower in NPI-3 tumours $(P=0.021$ compared with NPI-1 tumours; Fig. 4 , middle panel). No significant difference was seen for the Nov transcript between different groups (Fig. 4, right-hand panel; $P=0.07$, NPI-3 compared with NPI-1). Similar trends were seen when the target:CK19 ratio was assessed (Fig. 4, inserts)

Cyr61 was expressed at significantly higher levels in node-positive tumours compared with node-negative tumours ( $P=0.034$; Fig. 5, left-hand panel). Although node-positive tumours had higher levels of CTGF than node-negative ones (Fig. 5, middle panel), the difference was not statistically significant. No difference of Nov transcripts was seen between node-positive and -negative tumours (Fig. 5, right-hand panel). The same changes were also seen with the CCN:CK19 ratio (Fig. 5, inserts).

\section{Levels of $\mathrm{CCN}$ members and relationship with TNM staging and tumour differentiation}

Cyr61 was highly expressed in TNM-3/4 tumours (Fig. 6, left-hand panel), compared with TNM-1 tumours $(P=0.05$ and 0.106 respectively). A marginal and nonsignificant reduction of CTGF was seen in TNM-2/3/4 tumours, compared with TNM-1 tumours (Fig. 6, middle panel). The changes of Nov transcripts were similar to those of CTGF, except that TNM-4 tumours had significantly lower levels of Nov compared with TNM-1 tumours $(P=0.0048$; Fig. 6, right-hand panel). Similar trend of changes in each molecule were seen when the CCN:CK19 ratios were compared (Fig. 6, inserts).

Grade 2 and grade 3 tumours have significantly higher levels of Cyr61, compared with grade 1 tumours (Table 2). Although levels of CTGF and Nov in grade 3 tumours were generally low compared with grade 1 tumours, this difference is nonetheless not statistically significant (Table 2). 

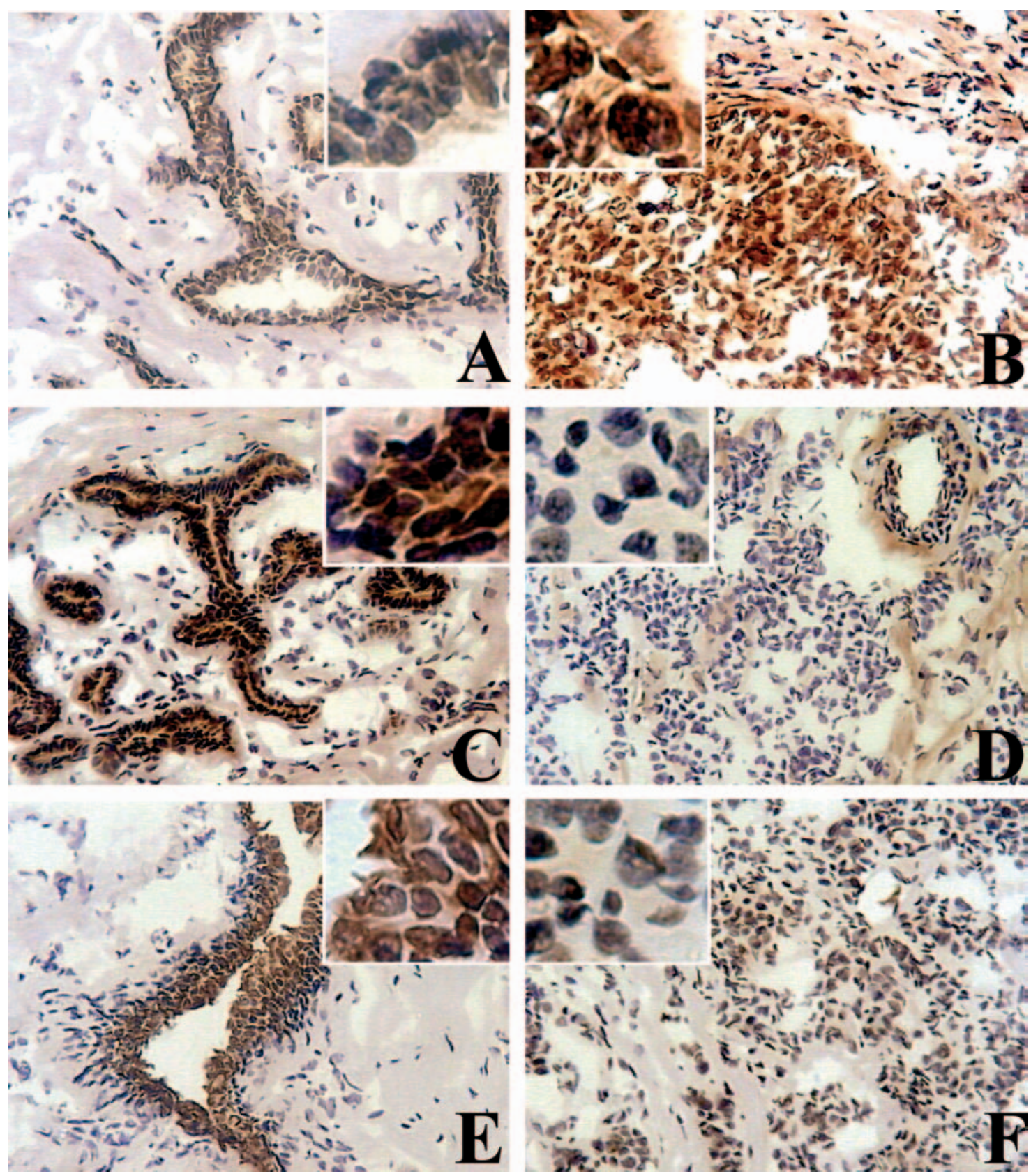

Figure 2 Immunohistochemical staining of Cyr61 (A, B), CTGF (C, D) and Nov (E, F) in normal (A, C, E) and tumour (B, D, F) tissues. Shown are magnifications $\times 100$ (main panels), and $\times 400$ (inserts).

\section{Correlation between levels of CCN members and ER status}

The possible relationship between $\mathrm{CCN}$ family members and ER status was also analysed. There was no significant correlation between ER and ER- $\beta$ with any of the family members when tumours were analysed as an entire cohort. However, ER was inversely correlated with Nov in NPI-3 tumours $(r=-0.32)$, and CTGF and Nov in TNM-3 tumours ( $r=-0.40$ and $r=-0.58$, respectively), and Nov in TNM-4 tumours $(r=-0.76)$. The correlations between ER- $\beta$ and the CCN members revealed an inverse correlation with all three members in tumours which developed metastasis $(r=-0.32$ for Cyr61, $r=-0.39$ for CTGF and $r=-0.49$ for Nov, respectively). 

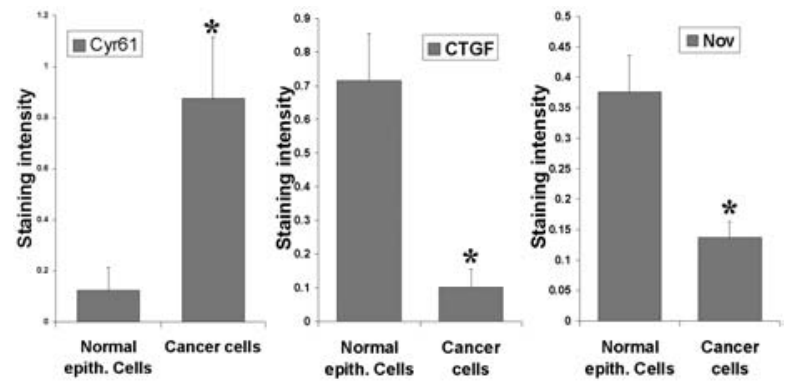

Figure 3 Staining intensity of normal mammary epithelial cells and breast cancer cells in mammary tissues. Cyr61 is shown on the left, CTGF in the middle and Nov on the right. Cytoplasmic staining of the respective molecules was analysed using Optimas 6 and is shown as relative staining intensity. ${ }^{*} P<0.05$ compared with normal epithelial cells.
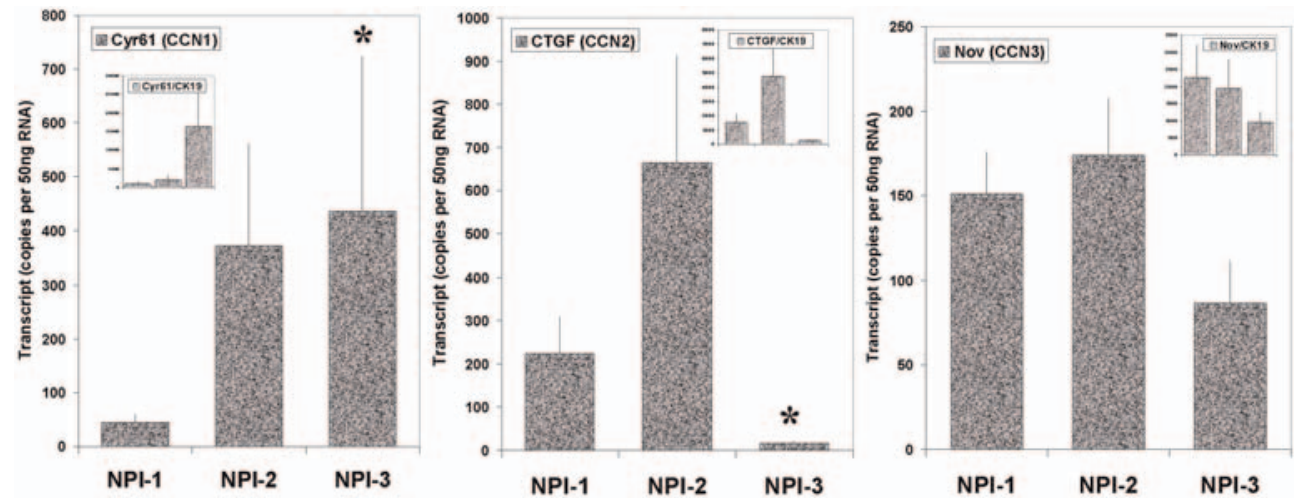

Figure 4 Expression of Cyr61 (left-hand panel), CTGF (middle panel), and Nov (right-hand panel) in relation to the Nottingham Prognostic Index (NPI), where NPI-1 (<3.4) indicates good prognosis, NPI-2 (3.4-5.4) is moderate and NPI-3 (> 5.4) is poor prognosis. ${ }^{*} P<0.02$ compared with $\mathrm{NPI}-1$. Inserts: respective $\mathrm{CCN}: \mathrm{CK} 19$ ratios.

\section{CCN and clinical outcome}

Following a 6-year follow up, patients were divided into four groups; those who remained disease-free, developed metastasis, had local recurrence and those who died of breast cancer (excluding deaths unrelated to breast cancer). In addition, the disease-free group was also compared with those groups with incidence (a combination of the other three groups). As shown in Fig. 7 (lefthand panel), patients with metastasis had significantly higher levels of Cyr61 $(P=0.016)$. A marginally high level of the molecule was also seen in those who had recurrence and mortality. The Cyr61:CK19 ratios were $193 \pm 120,3145 \pm 1311,31466 \pm 15670$ and $2951 \pm 1169$
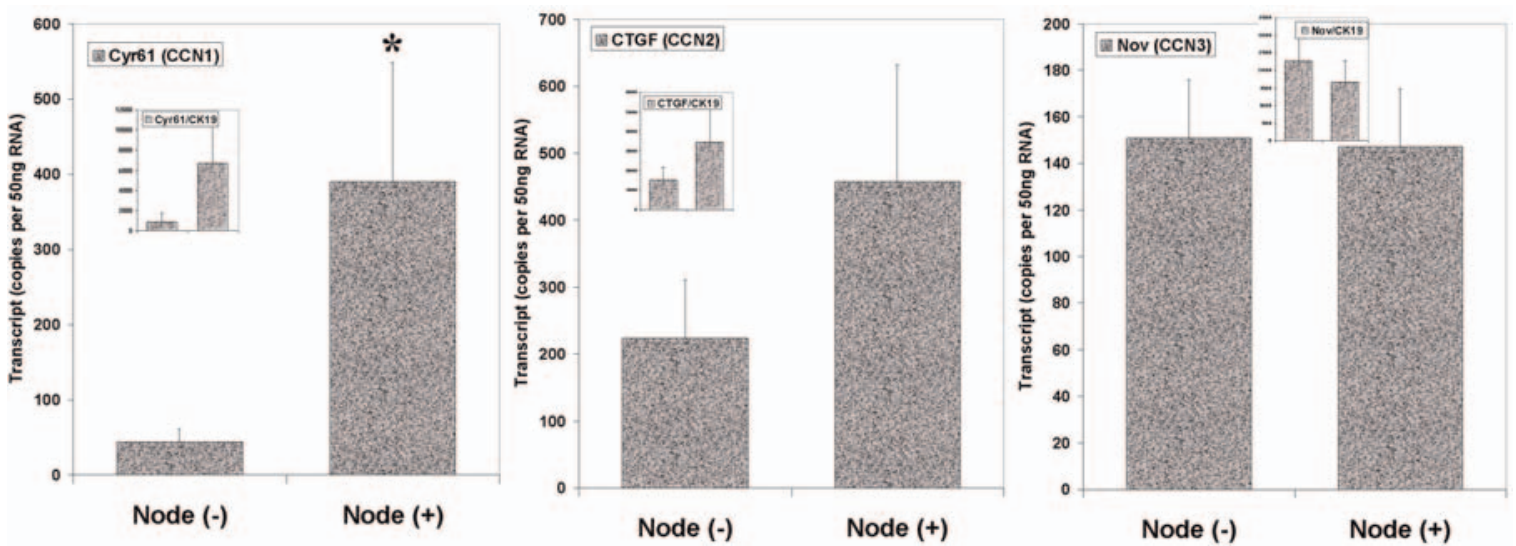

Figure 5 Levels of expression of the CCN family members in node-negative and node-positive tumours. Significantly high levels of Cyr61 were seen in node-positive tumours $\left({ }^{*} P=0.034\right)$. Inserts: respective CCN:CK19 ratios. 

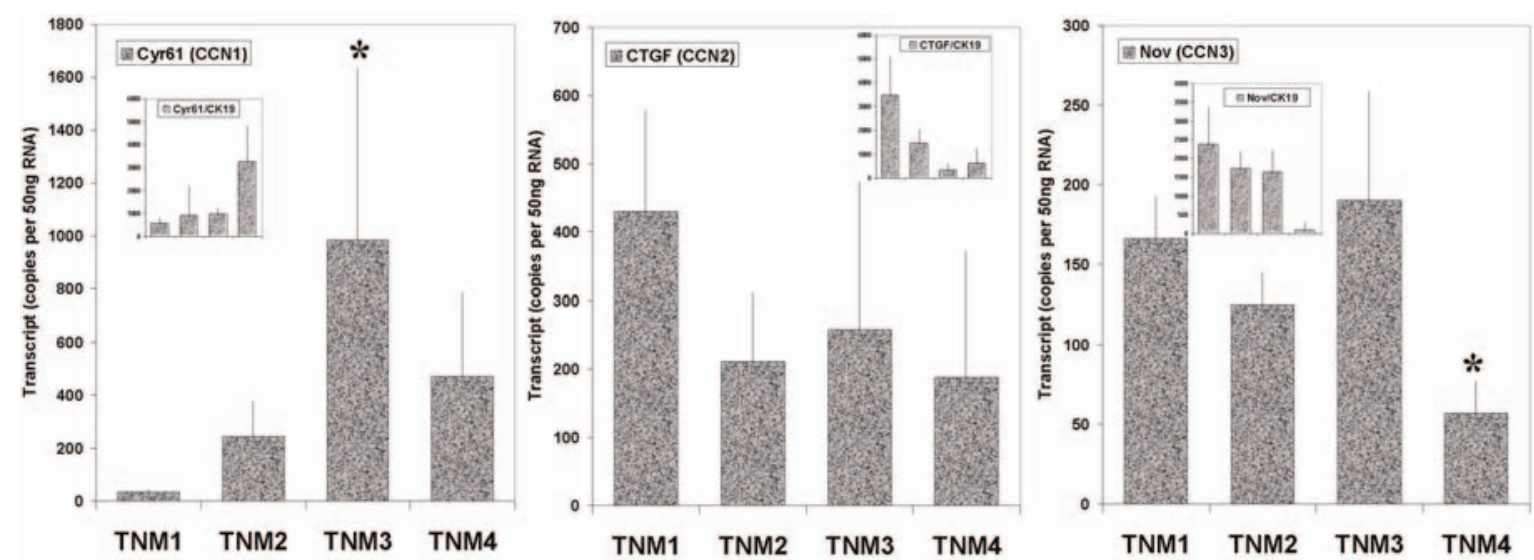

Figure 6 Levels of expression of Cyr61, CTGF and Nov in relation to TNM staging. Significantly higher levels of Cyr61 was seen in TNM-3 tumours $\left({ }^{*} P=0.05\right)$ and non-significant high level in TNM-4 tumours $(P=0.106)$ compared with TNM-1 tumours. Significantly lower levels of Nov were seen in TNM-4 tumours compared with TNM-1 $\left({ }^{*} P=0.0048\right)$. Inserts: respective CCN:CK19 ratios.

for those who remained disease free, developed metastasis, had local recurrence and died of breast cancer, respectively. The disease-free group had a significantly lower level than the other three groups combined $(P=0.0054$; Fig. 7, left-hand insert).

Levels of CTGF showed a very different trend to those of Cyr61, in which each group with incidence had a significantly lower level of CTGF compared with the disease-free group (Fig. 7, middle panel; $(P=0.012$, $P=0.0024$ and $P=0.0072$ in those with metastasis, local recurrence and who died of breast cancer compared with those remained disease free). The changes were also similarly reflected by the CTGF:CK 19 ratios, which were $3451 \pm 1283,26.3 \pm 11, \quad 522 \pm 393$ and $193 \pm 111$, for those who remained disease free, developed metastasis, had local recurrence and who died of breast cancer, respectively. As expected, the combination group (with complications) had a significantly lower level of CTGF than the disease-free group (Fig. 7, middle insert; $P=0.021$ ).

The only group that had a significantly lower level of Nov than the disease-free group was patients who died of

Table 2 Expression of CCN family members in different grades of breast tumours (data are expressed as mean \pm S.E.M. number of transcripts).

\begin{tabular}{lccc}
\hline \multicolumn{1}{c}{ Grade 1 } & Grade 2 & Grade 3 \\
\hline Cyr61 $13.5 \pm 9.5$ & $205 \pm 126$ & $315 \pm 145$ \\
& $(P=0.033$ vs grade 1$)$ & $(P=0.01$ vs grade 1$)$ \\
CTGF 664 \pm 348 & $134 \pm 52$ & $340 \pm 136$ \\
& & $(P=0.15$ vs grade 1$)$ & $(P=0.40$ vs grade 1$)$ \\
Nov $151 \pm 25$ & $174 \pm 34$ & $86.7 \pm 25$ \\
& & $(P=0.58$ vs grade 1$)$ & $(P=0.0307$ vs grade 1) \\
\hline
\end{tabular}

breast cancer $(P=0.024$; Fig. 7 , right-hand panel). The other groups and group combination failed to show a statistical difference from the disease-free group. This was true for both the transcripts and the Nov:CK19 ratio.

Kaplan-Meier survival and univariate analyses have shown that high levels of both $\mathrm{CTGF} / \mathrm{CCN} 2(P=0.033)$ and $\operatorname{Nov} / \mathrm{CCN} 3(P=0.0146)$ were significantly correlated with higher overall survival (Fig. 8). Higher levels of Cyr61, however, was associated with poorer survival, although this was statistically insignificant $(P=0.086)$.

\section{Discussion}

The current study has shown an aberrant expression of the CCN family members Cyr61, CTGF and Nov in human breast cancer. In addition to the clinical implications of aberrant expression, we also demonstrated a contrasting and differential expression of Cyr61 and CTGF in these patients.

The results on Cyr61 from our study concurs with a previous study by Xie et al. (2001a) on breast cancer in that Cyr61 is raised in breast tumours and is associated with aggressiveness of the tumours, including higher levels in NPI-3, nodal status, high grade, TNM-3 and tumours which developed metastasis. It would suggest that Cyr61 is a factor that is associated with a poor outcome in clinical tumours. Cyr61 has been shown to correlate with stage, tumour size and nodal status (Sampath et al. 2001, Xie et al. 2001a, 2001b). The study by Xie et al. did not provide information at the protein level. In clear contrast, Cyr61 has been shown to act as a tumour suppressor in non-small-cell lung cancer (Tong et al. 2001). In this study, Cyr61 has been shown to induce cell-cycle arrest at the G1 phase and human non-small-cell lung cancer tumours have far lower levels of Cyr61 compared with 

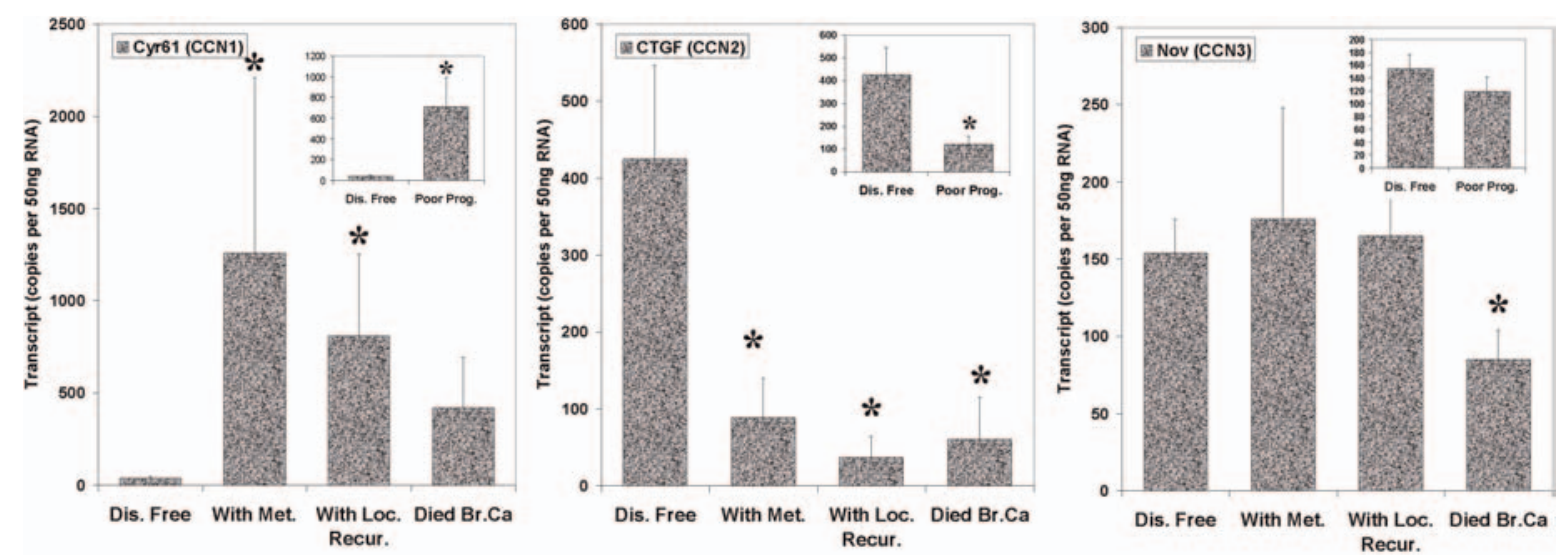

Figure 7 Levels of Cyr61 (left-hand panel), CTGF (middle panel) and Nov (right-hand panel) and clinical outcome over a 6-year follow-up period. Shown are patients who remained disease free, developed metastasis, had local recurrence and died of breast cancer. Inserts: comparison between those who remained disease free and those who developed complications (metastasis, recurrence and mortality). Statistical details are given in the text.

matched normal lung tissues. Expression of Cyr61 has been found to be reduced in thyroid cancer (Wasenius et al. 2003) as well as in prostate cancer tissues in which the reduction of Cyr61 appears to occur at both the protein and mRNA levels (Pilarsky et al. 1998). Cyr61 is overexpressed in highly invasive breast cancer cell lines compared with less-invasive cell lines (Xie et al. 2001b). It is possible therefore that the role of Cyr61 as a proaggressive factor or as a tumour suppressor is dependent on tumour histology.

Our data provide further insight into the differential expression pattern of the other $\mathrm{CCN}$ members, namely CTGF and Nov, from that of Cyr61. The levels of both molecules were lower in tumours at both the protein and mRNA levels. These two molecules are generally low in aggressive NPI-3 tumours (with predicted poor prognosis) and TNM-3/4 tumours. The significance of the low-level expression of CTGF is best reflected by the dramatically low level of expression in patients with a poor clinical outcome, including those who developed metastasis, recurrence and who died of breast cancer. A significant reduction of Nov is seen in patients who died of breast cancer (Fig. 7, right-hand panel), supported by significantly reduced survival (Fig. 8, bottom panel). The clear contrast between Cyr61 and CTGF/Nov suggests very different roles for the proteins of this family in the development of breast cancer. The mechanisms by which these three molecules acted differently are presently unclear. However, the observation made in this study is not isolated. Increased expression of CTGF has been shown in pancreatic cancer (largely in stromal cells) and is associated with the degree of fibrosis (Wenger et al. 1999). The CTGF gene promoter has been shown to be suppressed by the Wilms tumour suppressor WT1 in cell lines derived from Wilms tumours (Stanhope-Baker \& Williams 2000). Although CTGF was found to be overexpressed in oesophageal carcinoma (Koliopanos et al. 2002), highest levels were associated with longer survival in squamous cell carcinoma, but with shorter survival in adenocarcinoma. In squamous cell carcinoma of the oesophagus, high levels of CTGF are associated with longer survival (Koliopanos et al. 2002), and overexpression of CTGF in oral squamous cell carcinoma is associated with slower growth (Moritani et al. 2003). In breast cancer, CTGF has been found to be associated with nodal status and tumour size (Xie et al. 2001a). Adrenocortical tumours also have low levels of Nov compared with normal tissues and over-expression of Nov in these tumour cells would reduce the growth of glioma cells (Gupta et al. 2001, Martinerie et al. 2001). In a recent report, Nov has been found to be over-expressed in metastatic tumours and primary tumour with metastatic potential in rhabdomyosarcoma, Ewing's sarcoma (Manara et al. 2002). In breast cancer, Nov has been reported to have little correlation with clinical parameters (Xie et al. 2001a). It has been shown recently that Nov was reduced progressively from normal, benign tumour to malignant adrenocortical tissues (Martinerie et al. 2001). Thus, one of the possibilities is that in different tumours and cell types, different molecules may have differing roles, as suggested recently by Perbel (2004). CCN members including CTGF and Nov are known to have different variants, possibly as a result of alternative splicing (Perbel 2004). These variants may come into play in the overall biological functions of $\mathrm{CCN}$ members. It would be interesting to examine the expression of these variants, but this is beyond the scope of the current report. 

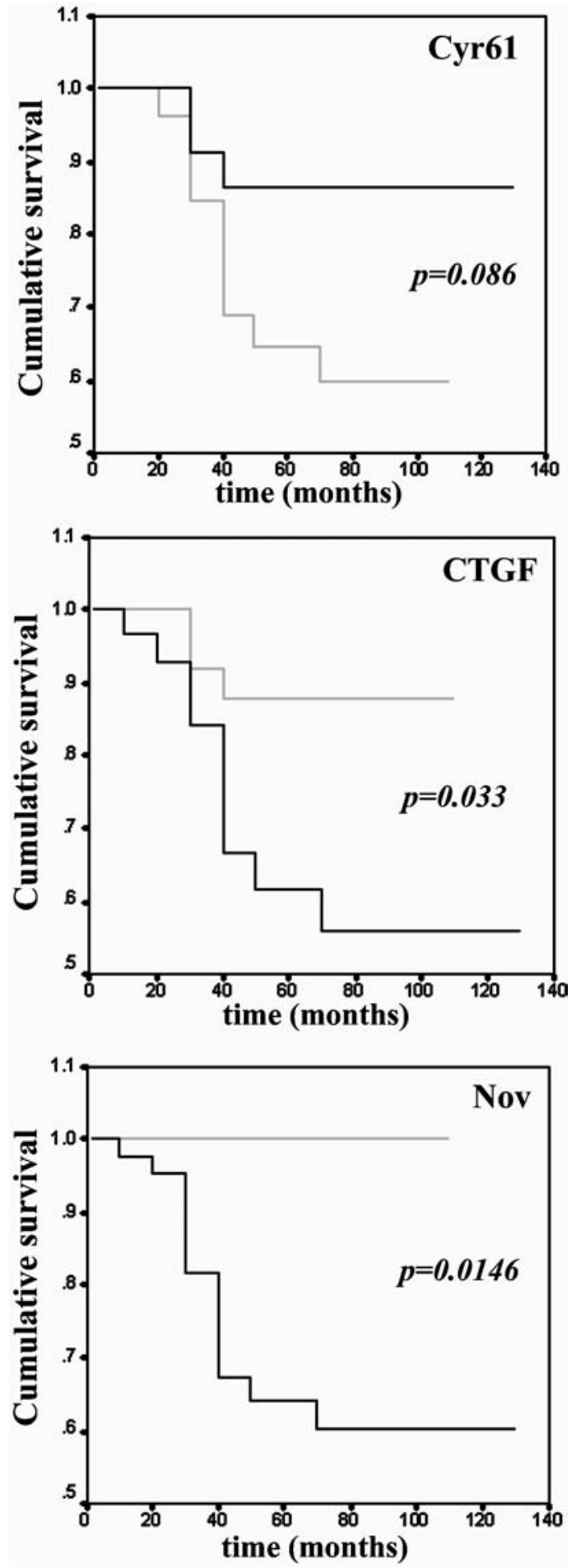

Figure 8 Levels of CCN family members and correlation with survival. Showing are Kaplan-Meier survival curves. Grey lines indicate high levels and black lines low levels of the respective CCN family member. Statistical testing was by univariate analysis; $P$ values are shown on the figure.
It has been shown that oestrogen is a powerful inducer of the expression of Cyr61 in breast cancer cells (Xie et al. 2001b). Expression of Cyr61 mRNA increased 8-12-fold in MCF-12A cells and 3-5-fold in MCF-7 cells after 24and 48-h exposure to oestrogen, respectively. Induction of Cyr61 mRNA can be blocked by tamoxifen and ICI182780, inhibitors of the ER (Xie et al. 2001b). In order to examine if a relationship exists between the status of ER (ER- $\alpha$ and ER- $\beta$ ) and the CCN family members, we have analysed the correlation coefficient between these molecules. It must be declared the correlation analyses of this nature are somewhat indirect and can only provide a general guide. ER- $\alpha$ and ER- $\beta$ are generally inversely correlated with the CTGF and Nov in aggressive tumours, such as TNM-3/4 and tumours with metastasis. No relationship, otherwise, has been found between ER and Cyr61. In addition, despite the fact that the CCN family has been found to interact with Notch-1 and S100A, and that these binding partners were aberrant in the same cohort of patients as reported from our recent studies (Parr and Jiang 2004, Jiang et al. 2004), no significant correlation was found between Cyr61, CTGF, Nov, Notch-1 and S100A4 (results not shown).

The current study has also provided information on potential use of CK19 as a means to address the possible difference in cellularity in different tissues. Although some discrepancies were observed in terms of the magnitude of the difference between the levels of transcripts based on equal amount of RNA and that based on the transcript:CK19 ratio, the two parameters generally agree with each other (Figs 1, 4, 5 and 6, main figures and respective inserts). This may suggest that CK19, when used in connection with epithelial markers, can be a useful tool in normalizing the differences in cellularity, as shown in our previous studies (King et al. 2004, Parr and Jiang 2004). On the other hand, the low degree of expression of these molecules in other cell types such as stromal and endothelial cells may have contributed to the minor discrepancies.

In summary, the $\mathrm{CCN}$ family members display different patterns of aberration in their expression in human breast cancer. Cyr61 is clearly linked to the aggressive nature of breast tumours. In clear contrast, CTGF, and to some degree Nov, show an inverse relationship with the aggressiveness of tumours, with lowest levels seen in patients with the poorest prognosis. The contrasting expression patterns of the $\mathrm{CCN}$ family may indicate a therapeutic and prognostic role for the family and also warrant further investigation into the molecular aspects of the aberrant expression of this pivotal family. We are currently investigating the role of the $\mathrm{CCN}$ family members in the growth and invasiveness of breast cancer cells. 


\section{Funding}

We would like to thank Breast Cancer Campaign for supporting our work (W G J).

\section{References}

Babic AM, Kireeva ML, Kolesnikova TV \& Lau LF 1998 CYR61, a product of a growth factor-inducible immediate early gene, promotes angiogenesis and tumor growth. PNAS $956355-6360$.

Brigstock DR 2003 The CCN family: a new stimulus package. Journal of Endocrinology 178 169-175.

Davies G, Jiang WG \& Mason MD 2000 Cell-cell adhesion and signalling intermediates in human prostate cancer. Journal of Urology 163 985-992.

Gupta N, Wang H, McLeod TL, Naus CC, Kyurkchiev S, Advani S, Yu J, Perbal B \& Weichselbaum RR 2001 Inhibition of glioma cell growth and tumorigenic potential by CCN3 (NOV). Molecular Pathology 54 293-299.

Hishikawa K, Oemar BS, Tanner FC, Nakaki T, Luscher TF \& Fujii T 1999 Connective tissue growth factor induces apoptosis in human breast cancer cell line MCF-7. Journal of Biological Chemistry 274 37461-37466.

Jiang WG, Watkins G, Lane J, Douglas-Jones A, Cunnick GH, Mokbel M \& Mansel RE 2003a Prognostic value of Rho familty and and rho-GDIs in breast cancer. Clinical Cancer Research 9 6432-6440.

Jiang WG, Douglas-Jones A \& Mansel RE 2003b Level of expression of PPAR-gamma and its co-activator (PPARGCA) in human breast cancer. International Journal of Cancer $106752-757$.

Jiang WG, Grimshaw D, Lane J, Martin TA, Parr C, Davies G, Laterra J \& Mansel RE 2003c Retroviral hammerhead transgenes to cMET and $\mathrm{HGF} / \mathrm{SF}$ inhibited growth of breast tumour, induced by fibroblasts. Clinical Cancer Research 9 4274-4281.

Jiang WG, Watkins G, Douglas-Jones A \& Mansel RE 2004 Psoriasin (S100A7) is aberrantly expressed in human breast cancer and is related to clinical outcomes. International Journal of Oncology 25 81-86.

King JAC, Ofori-Acquah AF, Stevens T, Al-Mehdi AB, Fodstad O \& Jiang WG 2004 Prognostic value of ALCAM in human breast cancer. Breast Cancer Research 6 R478-R487.

Koliopanos A, Friess H, di Mola FF, Tang WH, Kubulus D, Brigstock D, Zimmermann A \& Buchler MW 2002 Connective tissue growth factor gene expression alters tumor progression in esophageal cancer. World Journal of Surgery $\mathbf{2 6}$ 420-427.

Kothapalli D \& Grotendorst GR 2000 CTGF modulates cell cycle progression in cAMP-arrested NRK fibroblasts. Journal of Cellular Physiology 182 119-126.

Leu SJ, Lam SC \& Lau LF 2002 Pro-angiogenic activities of CYR61 (CCN1) mediated through integrins alphavbeta3 and alpha6beta 1 in human umbilical vein endothelial cells. Journal of Biological Chemistry 277 46248-46255.

Liu C, Liu XJ, Crowe PD, Kelner GS, Fan J, Barry G, Manu F, Ling N, De Souza EB \& Maki RA 1999 Nephroblastoma overexpressed gene (NOV) codes for a growth factor that induces protein tyrosine phosphorylation. Gene 238 471-478.

Manara MC, Perbal B, Benini S, Strammiello R, Cerisano V, Perdichizzi S, Serra M, Astolfi A, Bertoni F, Alami J, et al. 2002 The expression of ccn3(nov) gene in musculoskeletal tumors. American Journal of Pathology 160 849-859.

Martinerie C, Gicquel C, Louvel A, Laurent M, Schofield PN \& Le Bouc Y 2001 Altered expression of novH is associated with human adrenocortical tumorigenesis. Journal of Clinical Endocrinology and Metabolism 86 3929-3940.

Mo FE, Muntean AG, Chen CC, Stolz DB, Watkins SC \& Lau LF 2002 CYR61 (CCN1) is essential for placental development and vascular integrity. Molecular and Cellular Biology 22 8709-8720.

Moritani NH, Kubota S, Nishida T, Kawaki H, Kondo S, Sugahara T \& Takigawa M 2003 Suppressive effect of overexpressed connective tissue growth factor on tumor cell growth in a human oral squamous cell carcinoma-derived cell line. Cancer Letters 192 205-214.

Nazarenko IA, Bhatnagar SK \& Hohman RJ 1997 A closed tube format for amplification and detection of DNA based on energy transfer. Nucleic Acids Research 25 2516-2521.

Pan LH, Beppu T, Kurose A, Yamauchi K, Sugawara A, Suzuki M, Ogawa A \& Sawai T 2002 Neoplastic cells and proliferating endothelial cells express connective tissue growth factor (CTGF) in glioblastoma. Neurology Research $\mathbf{2 4}$ $677-683$.

Parr C \& Jiang WG 2004 The Notch receptors, Notch-1 and Notch-2, in human breast cancers. International Journal of Molecular Medicine (In Press).

Perbel B 2004 CCN proteins: multifunctional signalling regulators. Lancet 363 62-64.

Pilarsky CP, Schmidt U, Eissrich C, Stade J, Froschermaier SE, Haase M, Faller G, Kirchner TW \& Wirth MP 1998 Expression of the extracellular matrix signaling molecule Cyr61 is downregulated in prostate cancer. Prostate 36 85-91.

Sampath D, Winneker RC \& Zhang Z 2001 Cyr61, a member of the $\mathrm{CCN}$ family, is required for MCF-7 cell proliferation: regulation by 17 beta-estradiol and overexpression in human breast cancer. Endocrinology 142 2540-2548.

Sampath D, Winneker RC \& Zhang Z 2002 The angiogenic factor Cyr61 is induced by the progestin R5020 and is necessary for mammary adenocarcinoma cell growth. Endocrine 18 147-159.

Stanhope-Baker P \& Williams BR 2000 Identification of connective tissue growth factor as a target of WT1 transcriptional regulation. Journal of Biological Chemistry $\mathbf{2 7 5}$ 38139-38150.

Tong X, Xie D, O'Kelly J, Miller CW, Muller-Tidow C \& Koeffler HP 2001 Cyr61, a member of CCN family, is a tumor suppressor in non-small cell lung cancer. Journal of Biological Chemistry 276 47709-47714.

Tsai MS, Hornby AE, Lakins J, Lupu R 2000 Expression and function of CYR61, an angiogenic factor, in breast cancer cell lines and tumor biopsies. Cancer Research 60 5603-5607.

Tsai MS, Bogart DF, Castaneda JM, Li P \& Lupu R 2002a Cyr61 promotes breast tumorigenesis and cancer progression. Oncogene 21 8178-8185. 
Tsai MS, Bogart DF, Li P, Mehmi I, Lupu R $2002 b$ Expression and regulation of Cyr61 in human breast cancer cell lines. Oncogene 21 964-973.

Wasenius VM, Hemmer S, Kettunen E, Knuutila S, Franssila K \& Joensuu H 2003 Hepatocyte growth factor receptor, matrix metalloproteinase-11, tissue inhibitor of metalloproteinase-1, and fibronectin are up-regulated in papillary thyroid carcinoma: a cDNA and tissue microarray study. Clinical Cancer Research 9 68-75.

Wenger C, Ellenrieder V, Alber B, Lacher U, Menke A, Hameister H, Wilda M, Iwamura T, Beger HG, Adler G, Gress TM 1999 Expression and differential regulation of connective tissue growth factor in pancreatic cancer cells. Oncogene 18 1073-1080.
Xie D, Nakachi K, Wang H, Elashoff R \& Koeffler HP $2001 a$ Elevated levels of connective tissue growth factor, WISP-1, and CYR61 in primary breast cancers associated with more advanced features. Cancer Research 61 8917-8923.

Xie D, Miller CW, O'Kelly J, Nakachi K, Sakashita A, Said JW, Gornbein J \& Koeffler HP 2001b Breast cancer. Cyr61 is overexpressed, estrogen-inducible, and associated with more advanced disease. Journal of Biological Chemistry 27614187 14194.

Ye L, Martin TA, Parr C, Harrison G, Mansel RE \& Jiang WG 2003 Biphasic effects of $17-\beta$-oestradiol on expression of occludin and transendothelial resistance (TER) and paracellular permeability in human vascular endothelial cells. Journal of Cellular Physiology 196 362-369. 